\title{
Gattungsgeschichte als Problemgeschichte
}

\author{
Am Beispiel des carmen heroicum des 17. Jahrhunderts im \\ Allgemeinen und von Christian Ulrich Illenhöfers Poetischer \\ Beschreibung über die zweite Schlacht bei Breitenfeld (1643) \\ im Besonderen
}

\section{Fallbeispiel: Christian Ulrich Illenhöfer}

Im Jahr 1643 erschien bei einer namentlich nicht bekannten Offizin in Stockholm eine Poetische Beschreibung Der Denckwürdigen Blutigen Schlacht/So den 23. Octobris | 2. Novembris Anno 1642. Jm Breiten-Felde Nahe Leipzig/Zwischen Dem Käyserl. General-Feldmarschall Graff Piccolomini/ vnd Dem Schwedischen General-Feldmarschall Herrn Linnardt Torstenson vorgangen/In welcher die Käyserl. den Kürtzern gezogen [...]. ${ }^{1}$ Als Autor firmiert ein Christian Ulrich Illenhöfer, der sich als Student der Rechte ausweist. Thema des Texts ist die Schlacht bei Breitenfeld. Hiervon hat es im Dreißigjährigen Krieg zwei gegeben. Die erste Schlacht bei Breitenfeld war 1631 zwischen den Truppen Gustavs II. Adolf von Schweden und jenen der katholischen Liga unter Führung Johann T’Serclaes von Tillys ausgetragen und von den Schweden gewonnen worden. Diese Schlacht hatte eine eminente Bedeutung für den Kriegsverlauf, denn sie führte, in den Worten Herfried Münklers, "zum Zusammenbruch der kaiserlich-ligistischen Macht in Norddeutschland «. ${ }^{2}$ Die erste Schlacht bei Breitenfeld kann aber, wie der Titel des vorliegenden Texts lehrt, hier nicht gemeint sein, sondern es muss um die zweite Schlacht bei Breitenfeld gehen, die 1642, wieder zwischen den schwedischen und den kaiserlichen Truppen, ausgetragen wurde. Gustav Adolf und Tilly waren inzwischen beide gefallen, ebenso wie der dritte große Feldherr

1 Christian Ulrich Illenhöfer: Poetische Beschreibung Der Denckwürdigen Blutigen Schlacht/ So den 23. Octobris | 2. Novembris Anno 1642. Jm Breiten-Felde Nahe Leipzig/ Zwischen Dem Käyserl. General-Feldmarschall Graff Piccolomini/ vnd Dem Schwedischen General-Feldmarschall Herrn Linnardt Torstenson vorgangen/ In welcher die Käyserl. den Kürtzern gezogen [...]. Stockholm 1643.

2 Herfried Münkler: Der Dreißigjährige Krieg. Europäische Katastrophe, Deutsches Trauma 1618-1648. Berlin 2017, S. 758.

Dirk Werle, Heidelberg 
der mittleren Kriegsphase, Albrecht von Wallenstein; Heerführer waren diesmal Ottavio Piccolomini auf Seiten der kaiserlichen und Linnardt Torstenson auf Seiten der schwedischen Truppen. Dass diese Schlacht am selben Ort wie jene elf Jahre zuvor, auf einer Ebene nordwestlich von Leipzig, stattfand, war einesteils den Kontingenzen der Kriegsentwicklung geschuldet, hatte andernteils sicher damit zu tun, dass Kursachsen als protestantischer Bündnispartner des Kaisers, das nur zwischen 1631 und 1635 vorübergehend auf die Seite der Schweden überlief, innerhalb dieser Entwicklung immer wieder eine exponierte Position erhielt; es hatte in der konkreten Situation aber vor allem - so wiederum Münkler - sicher auch seine Ursachen in symbolpolitischen Erwägungen sowie in kriegsstrategischem Kalkül: Der schwedische Heerführer Torstenson wollte sich vermutlich, indem er seine Truppen fast am selben Ort aufmarschieren ließ wie 1631 Gustav Adolf, in die Tradition dieses siegreichen Feldherrn und gerade auch nach seinem Tode gefeierten Heros der protestantisch-schwedischen Sache stellen, dadurch auch seine Soldaten an einen zweiten Sieg glauben machen und gegenüber der Öffentlichkeit eine Kontinuität schwedischer Überlegenheit im Krieg behaupten. ${ }^{3}$ Außerdem war die Ebene bei Breitenfeld, die durch einen Wald in der Mitte geteilt wurde, schlachtstrategisch günstig gelegen, wie man im Detail ebenfalls bei Münkler nachlesen kann. ${ }^{4}$ Die zweite Schlacht bei Breitenfeld wurde wieder von den Schweden gewonnen; sie ging darüber hinaus auch als eine der blutigsten Schlachten des Dreißigjährigen Krieges in die Geschichte ein, und sie besaß eine große Bedeutung für die Gestaltung der Nachkriegsordnung, indem sie kurz vor der Aufnahme der Friedensverhandlungen in Münster und Osnabrück die kriegerische Ausgangslage bedeutend zugunsten der protestantischen Seite verschob.

Da der vorliegende Text in Stockholm veröffentlicht worden ist, ist von vornherein klar, für welche Kriegspartei der Autor optiert, und es wird auch bereits im Titel deutlich durch die umgangssprachliche Formulierung, die Kaiserlichen hätten bei der Schlacht »den Kürtzern gezogen «. ${ }^{5}$ Bemerkenswert ist in diesem Zusammenhang aber, dass ebenfalls bereits im Titel für die Schlacht zwei alternative Daten angegeben werden, der 23. Oktober und der 2. November 1642. Der Hintergrund hierfür ist, dass 1582 Papst Gregor XIII. den später so genannten gregorianischen Kalender eingeführt hatte, der daraufhin in den meisten katholischen Territorien Europas verwendet wurde. Die protestantischen Territorien des Reichs und Europas behielten aus ideologischen Gründen gro-

3 Vgl. ebd., S. 759.

4 Ebd., S. 759 f.

5 Allgemein fällt auf, dass der Text häufig punktuell durch umgangssprachliche Formulierungen das hohe Stilniveau, das in der Zeit für eine epische Versdichtung gefordert war, unterbietet. Ob man dem Autor hier eine Intention unterstellen darf, sei dahingestellt. 
ßenteils noch lange den bis dahin maßgeblichen julianischen Kalender bei und holten die Kalenderreform erst im 18. Jahrhundert nach. ${ }^{6}$ Indem der protestantische Autor für die Schlacht bei Breitenfeld nicht nur, wie erwartbar, das Datum des julianischen Kalenders angibt, sondern alternativ auch jenes des gregorianischen, deutet er an, dass er nicht nur protestantische Leser adressiert, sondern deutsche Leser aller Konfessionen. Bereits im Titel zeigt sich mithin eine Spannung, die, wie wir sehen werden, den gesamten Text durchzieht und über deren Deutung man sich als Interpret Gedanken machen muss: Der Autor ergreift für die protestantische Sache Partei, adressiert dabei aber alle Deutschen, und die Katholiken durchaus nicht in polemischer Absicht, sondern mit inkludierendem Gestus. Vor diesem Hintergrund wird auch die umgangssprachliche Formulierung im Titel des Texts nochmals anders verständlich: Die Wendung >den Kürzeren ziehen` verweist auf die Entscheidungsmethode des Losens mit unterschiedlich langen Stöckchen. Diese Entscheidungsmethode verweist die in Frage stehende Entscheidung an das Schicksal oder den Zufall. Insofern in der Redewendung unbestimmt bleibt, ob die Niederlage der Kaiserlichen ein vorherbestimmtes Schicksal oder ein Resultat historischer Kontingenz ist, ist bereits im Titel angedeutet, dass die Haltung des Autors und des epischen Sprechers nicht ungebrochen ist: Zwar ist der Autor und epische Sprecher als Protestant auf Seiten der Schweden, aber als Deutscher nimmt er eine empathische Haltung auch gegenüber den besiegten Kaiserlichen ein.

Der Text enthält eine an General Torstenson adressierte Widmungsvorrede, in der der Autor Illenhöfer diesen als seinen "gnädigen Herrn « anspricht ${ }^{7}$ und rekapituliert beziehungsweise für einen Sekundäradressaten überhaupt erst darlegt, wie er in schwedische Dienste geraten und dazu gekommen ist, das vorliegende Gedicht zu schreiben. Diese Ausführungen sind für den Literaturhistoriker besonders wertvoll, denn aus ihnen erfährt man einiges über den Autor, über den, soweit jedenfalls mein bisheriger Ermittlungsstand, sonst nichts in Erfahrung zu bringen ist: Laut Verzeichnis der im deutschen Sprachraum erschienenen Drucke des 17. Jahrhunderts existieren vom selben Verfasser keine weiteren Schriften, und über seine Person findet man weder im Killy oder im Dünnhaupt, noch in ADB oder NDB, noch in Zedler oder Jöcher einschlägige Informationen, und auch eine Recherche im World Biographical Information System bleibt erfolglos. Erwähnung findet der Autor in Volker Meids großer

6 Vgl. für den Hintergrund Achim Landwehr: Geburt der Gegenwart. Eine Geschichte der Zeit im 17. Jahrhundert. Frankfurt a.M. 2014, S. 263-270.

7 Illenhöfer: Poetische Beschreibung (s. Anm. 1), Bl. A $2^{\mathrm{r}}$. 
Geschichte der Deutschen Literatur im Zeitalter des Barock, aber lediglich im Zuge einer sehr kursorischen Inhaltsangabe der Poetischen Beschreibung der Schlacht bei Breitenfeld. ${ }^{8}$ Aus deren Vorrede erfährt man immerhin, dass der Autor aus Colberg stammt - unklar ist jedoch, ob Colberg in Thüringen oder jenes in Pommern gemeint ist -, dass er in Straßburg die Rechte studiert hat ${ }^{9}$ und 1641 in den Wirren des Krieges zunächst in schwedische Gefangenschaft und dann in die Dienste Torstensons geraten ist. ${ }^{10}$ Mit Blick auf seine poetische Schlachtbeschreibung beansprucht Illenhöfer in der Vorrede die Rolle eines Kriegsberichterstatters, der einerseits beschreiben wolle, was er »mit Augen selbst gesehen «, und andererseits referieren wolle, was er »von anderen [...] erforschet und aufgezeichnet « habe. ${ }^{11}$ Illenhöfer beruft sich also auf Autopsie und auf investigative Recherche. Die so gewonnenen Nachrichten habe er, so Illenhöfer weiter, "in teutsche Verse bringen « wollen, ${ }^{12}$ er zielt also mit seinem Text explizit den Bereich der deutschsprachigen Dichtung an. Dichtung soll, so war man noch in der frühen Neuzeit überzeugt, einerseits die Leser belehren und andererseits unterhalten, und dieses Ziel artikuliert Illenhöfer in rhetorisch ingeniöser Wendung verknüpft mit einem zweiten, der dichterischen Rühmung General Torstensons als großen Kriegshelden, wenn er diesem als Widmungsadressaten ansinnt, er möge »zu Uberlesung« seines Gedichts »nur so viel stunden anwenden/ als Ew. Exc. Zeit bedurffte die Käyserl. Armee im breiten Felde von ihren gefassten Kampffplatze wegzuschlagen ${ }^{13}{ }^{13}$ Soll erstens heißen, das Gedicht soll möglichst kurzweilig zu lesen sein, und zweitens, General Torstenson ist als Kriegsheld seinen Gegnern so überlegen, dass er nur kurze Zeit braucht, um sie zu besiegen.

8 Volker Meid: Die deutsche Literatur im Zeitalter des Barock. Vom Späthumanismus zur Frühaufklärung 1570-1740. München 2009, S. 524. Eine beiläufige Erwähnung des Autors und seines Texts im Kontext einer Gattungsgeschichte epischer Versdichtungen im 17. Jahrhundert findet sich bereits bei Emil Stern: Das deutsche Epos des 17. Jahrhunderts (1. Theil). In: Programm der deutschen k.k. Staats-Realschule in Budweis, veröffentlicht am Schluss des Schuljahres 1895, Budweis 1895, S. 1-26 [der zweite Teil der Abhandlung erschient im Schulprogramm derselben Schule ein Jahr später, S. 1-27], hier S. 15. Illenhöfer und sein Text werden hier als Teil einer Gruppe epischer Versdichtungen abgehandelt, »welche Zeitereignisse behandeln" (ebd., S. 14) und deren gemeinsames Merkmal nach Stern »die Scheu vor wirklicher erzählender Darstellung der Thatsachen« (S. 15) sei.

9 In den Matrikeln der Universität Straßburg findet sich sein Name jedoch nicht; vgl. Die alten Matrikeln der Universität Straßburg 1621 bis 1793. Bearbeitet von Gustav C. Knod. Dritter Band: Personen- und Ortsregister. Straßburg 1902.

10 Illenhöfer: Poetische Beschreibung (s. Anm. 1), Bl. A $2^{\mathrm{r}}$ f.

11 Ebd., Bl. A $3^{\mathrm{r}}$.

12 Ebd.

13 Ebd. 
Der Haupttext der Poetischen Beschreibung ist in paargereimten (im Druck mit Hilfe eines Zeilenzählers durchgezählten) Alexandrinern abgefasst, zu der Zeit auch >heroische Verse، genannt, und beginnt mit einer Bitte des Dichters an den Gott Apoll, ihm die Kraft zu verleihen, das »Lob viel edler Krieges-Helden « und insbesondere des »andere[n] Gustav« Linnardt Torstenson zu singen. ${ }^{14}$ Es wird geschildert, wie den Dichter als Resultat des Anrufs Apollos und durch dessen Hilfe der furor poeticus erfasst und die Inspiration durchströmt: »Jetzt fühl ich gleich in mir | Den angezündten Geist/ die fewrige Begier/ | Das Werck zu treten an. Der Kiel beginnt zu lauffen/ | Es kommen gleich die Wort aus jhme voller Hauffen | Geflossen aufs Papier.« ${ }^{15}$ Damit der Strom nicht versiegt, ruft der Dichter gleich noch eine andere Gottheit um Beistand an, Fama, die Göttin des Ruhms und der dichterischen Kunde. Ein Gedicht in heroischen Versen, das Helden besingt und mit einem Anruf an eine Gottheit mit der Bitte um Inspiration beginnt - bei einem solchen Text wussten zeitgenössische Leser sofort, um welches dichterische Genre es sich handelte. Der Text ist erkennbar konzipiert und ausgeführt als carmen heroicum, als epische Versdichtung. Vor diesem Hintergrund wird auch die in der Vorrede artikulierte Intention, in dichterischer Form die historische Wahrheit künden zu wollen, nochmals anders verständlich, denn genau das galt in der Zeit ebenfalls als Charakteristikum eines carmen heroicum. ${ }^{16}$ Carmina heroica galten in der zeitgenössischen Poetik als dichterische Königsdisziplin, ${ }^{17}$ doch spätestens seit Johann Christoph Gottscheds einflussreichen Ausführungen in der Critischen Dichtkunst waren die Gelehrten vom 18. bis zum 20. Jahrhundert meistenteils der Ansicht, es habe im 17. Jahrhundert nur wenige epische Versdichtungen im deutschen Kulturraum gegeben, ${ }^{18}$ ja,

14 Ebd., Bl. A 3 $3^{\text {v }, ~ V . ~} 3$ und 6. Eine ausführlichere Untersuchung wären die den Text vielgestaltig kommentierenden Marginalien wert.

15 Ebd., V. 9-13.

16 Vgl. Dirk Werle: Von hohem Wesen. Zu Wahrheitsanspruch und Gattungspoetik epischer Versdichtungen im 17. Jahrhundert (am Beispiel von Caspar von Barth und Georg Greflinger). In: Zeitschrift für Germanistik N.F. 28 (2018), S. 10-24.

17 Vgl. etwa die einschlägige Darstellung bei Martin Opitz: Buch von der Deutschen Poeterey (1624). Studienausgabe. Hg. von Herbert Jaumann. Stuttgart 2002, S. 26-30.

18 Gottsched behandelt in der Critischen Dichtkunst die Gattung Epos ausführlich im Kapitel »Von der Epopee oder dem Heldengedichte«, geht dabei aber vor allem auf die antiken Texte ein. Aus dem Bereich der zeitgenössischen deutschen Literaturgeschichte erwähnt er Wolf Helmhardt von Hohbergs 1663/64 in Leipzig erschienenen, monumentalen Habspurgischen Ottobert sowie Christian Heinrich Postels 1724 postum in Hamburg veröffentlichten Großen Wittekind und kommentiert: "Diese Fabeln an sich, oder die Gedichte selbst sind besser gerathen, als ihre rauhe und garstige Verse: daher sich sehr wenige überwinden können, solche verdrießliche Werke zu lesen. « Johann Christoph Gottsched: Versuch einer critischen Dichtkunst: Anderer besonderer Theil. Berlin und New York 1973 (Ausgewählte Werke. Hg. von Joachim 
gelegentlich wurde gar vom »Scheitern des epischen Projekts« im 17. Jahrhundert gesprochen. ${ }^{19}$ Gottscheds Behauptung beruht aber auf einem Irrtum (bei dem nicht ganz sicher ist, ob er dem Autor nicht vielleicht halb intendiert unterlaufen ist), und die These vom Scheitern beruht auf einer fehlgeleiteten Interpretation; im Gegenteil ist die Gattungsgeschichte in der Zeit ausgesprochen vielgestaltig. Insbesondere der Dreißigjährige Krieg und seine Protagonisten werden immer wieder Gegenstand epischer Versdichtungen in deutscher wie in lateinischer Sprache, sei es philosophisch in Martin Opitz' 1633 erschienenen Trostgedichten in Widerwärtigkeit des Krieges und in verschiedenen anderen carmina heroica desselben Autors, sei es allegorisch in Jacob Baldes 1637 veröffentlichter $\mathrm{Ba}$ trachomyomachia tuba Romana cantata oder auch in Andreas Gryphius' ebenfalls 1637 erschienenem Olivetum, sei es chronikalisch in Georg Greflingers 1653 publizierter Reimchronik Der Deutschen Dreißigjähriger Krieg sowie in vielen weiteren Texten. ${ }^{20}$ Illenhöfers Poetische Beschreibung der zweiten Schlacht bei Breitenfeld ist in dieser Reihe zu situieren.

Birke und Brigitte Birke, Bd. 6, 2) [als Grundlage der Edition dient die dritte Auflage von 1742], S. 279-308 (»Von der Epopee oder dem Heldengedichte«), hier S. 291.

19 Vgl. den Titel des Schlusskapitels bei Ernst Rohmer: Das epische Projekt. Poetik und Funktion des >carmen heroicum` in der deutschen Literatur des 17. Jahrhunderts. Heidelberg 1998. Gerechter Weise ist hervorzuheben, dass Rohmers Ausführungen deutlich komplexer und dem Gegenstand angemessener sind, als es der zitierte Titel des Schlusskapitels seines Buchs vermuten lässt.

20 Martin Opitzen Trostgedicht In Widerwertigkeit Deß Kriegs. In vier Bücher abgetheilt/ Und vor etlichen Jahren anderwerts geschrieben. In: ders.: Geistliche Poemata 1638. Hg. von Erich Trunz. Tübingen 1966, S. 334-408; Jacob Balde und seine Batrachomyomachia. Text, Übersetzung und Kommentar von Peter Mathes. 2 Bde. Heidelberg 2020; Andreas Gryphius: Olivetum. In: Ders.: Herodes. Der Ölberg. Lateinische Epik. Hg., übersetzt und kommentiert von Ralf Georg Czapla. Berlin 1999, S. 150-257; Georg Greflinger: Der Deutschen Dreyßig-Jähriger Krieg 1657. Hg. von Peter Michael Ehrle. München 1983. Vgl. zu Opitz’ Trostgedicht und Greflingers Dreyßig-Jährigem Krieg Dirk Werle: Knowledge in Motion between Fiction and Non-Fiction. Epic Poems and Didactic Poetry in the Seventeenth Century (Martin Opitz and Georg Greflinger). In: Daphnis 45 (2017), S. 563-577; zu Gryphius' Olivetum ders.: Andreas Gryphius' Olivetum und die Traditionen des carmen heroicum im siebzehnten Jahrhundert. In: Oliver Bach, Astrid Dröse (Hg.): Andreas Gryphius (1616-1664). Zwischen Tradition und Aufbruch. Berlin, Boston 2020, S. 311-327; zu Baldes Batrachomyomachia ders. und Katharina Worms: Jacob Baldes Batrachomyomachia Homeri Tuba Romana cantata (1637) und der Dreißigjährige Krieg. In: Scientia Poetica 22 (2018), S. 214-228, sowie die jeweils in diesen Beiträgen zitierte Forschungsliteratur. Zu Opitz' weiteren carmina heroica vgl. darüber hinaus Dirk Werle: Das carmen heroicum und der Krieg. Martin Opitz' Ratispona in libertatem vindicata (1633). In: Daphnis 47 (2019), S. 238-254; Marie-Thérèse Mourey: Historizität und Fiktion in Martin Opitzens Versepen. Ebd., S. 221-237; Jörg Robert: Poetische Naturwissenschaft. Martin Opitz' Lehrgedicht Vesuvius (1633). In: Daphnis 46 (2018), S. 188-214. Zu carmina heroica des 17. Jahrhunderts im Allgemei- 
Der Götteranruf zu Beginn des Texts wächst sich genregemäß zu einem veritablen Proömium aus, in dem der Dichter sich selbst in spezifischer Weise charakterisiert: Er ist »von Teutschem Blut«, sein Herz ist »[m]it alter Redlichkeit» voll, er spricht stets die »Warheit«: »Weiß hab ich allzeit weiß/ vnd schwartzes schwartz genannt «, schreibt er. ${ }^{21}$ Verstellung ist ihm fremd, er nennt sich einen Feind der Lügen ${ }^{22}$ und nimmt sich vor, das, was er zu sagen hat, auf »alt teutsch « und »in hoch-Teutsch», also in einem Deutsch, wie man es in den südlicheren Regionen des deutschen Kulturraums sprach, vorzutragen. ${ }^{23}$ Hier wird eine in der Zeit geläufige Vorstellung artikuliert, wonach Redlichkeit eine altdeutsche Tugend ist, die allenfalls durch den Einfluss anderer Nationalkulturen in Gefahr gerät, und eine gepflegte deutsche Sprache als korrelierend mit einer intakten moralischen Verfassung gesehen wird. ${ }^{24}$ Diese Position wurde in den 1640er Jahren nicht zuletzt von Autoren wie Jesaias Rompler von Löwenhalt und Johann Michael Moscherosch im Umfeld der Straßburger Tannengesellschaft vertreten, ${ }^{25}$ und in Straßburg hatte Illenhöfer ja ausweislich der Vorrede seines Gedichts studiert.

Illenhöfer greift, um seiner epischen Schlachtbeschreibung den geeigneten Rahmen zu geben, auf Traditionen einer bestimmten Spielart des carmen heroicum zurück, des Bibelepos nämlich. Er baut ganz entsprechend den Konventionen der antiken Epik neben beziehungsweise über der Ebene menschlicher Akteure eine zweite Akteursebene auf, jene der Götterwelt. Da aber im Rahmen eines monotheistischen Weltbildes nicht viele Götter auf den unterschiedlichen Seiten am Kampf teilnehmen können, lässt er Gott und die himmlischen Heer-

nen und im Dreißigjährigen Krieg im Besonderen die Schwerpunkthefte Dirk Werle (Hg.): Erforschung von epischen Versdichtungen im langen 17. Jahrhundert (ca. 1570-1740). In: Zeitschrift für Germanistik N.F. 28 (2018), S. 7-88; Uwe Maximilian Korn u. a. (Hg.): Das carmen heroicum in der frühen Neuzeit. In: Daphnis 46 (2018), S. 1-326; Sylvia Brockstieger, Dirk Werle (Hg.): Erzählen zwischen Realität und Fiktion. Der Dreißigjährige Krieg in epischen Versdichtungen und erzählender Prosa des 17. Jahrhunderts. In: Scientia Poetica 22 (2018), S. 209-298.

21 Illenhöfer: Poetische Beschreibung (s. Anm. 1), Bl. A 4 ${ }^{\mathrm{r}}$, V. 9-13.

22 Ebd., V. 15: „Der Lügen bin ich feind.«

23 Ebd., V. $20 \mathrm{f}$.

24 Vgl. in diesem Sinne auch das Spiel mit den Wörtern `teutsch Stelle im Text, wo der Dichter klagt: "So seyn wir mehr nicht teutsch/ indem wir also teuschen/ | Wo ist/ O Teutschland/ nun dein alte Redlichkeit/ | Dein Trew-auffrichtig-seyn?« Ebd., Bl. E 1.

25 Vgl. Wilhelm Kühlmann, Walter E. Schäfer: Literatur im Elsaß von Fischart bis Moscherosch. Gesammelte Studien. Tübingen 2001, vor allem S. 97-159 [»Die Tannengesellschaft eine Straßburger literarische Sozietät«] und S. 161-174 [»Moscherosch und die Sprachgesellschaften des 17. Jahrhunderts - Aspekte des barocken Kulturpatriotismus«]. 
scharen auf Seiten der Schweden in das Geschehen eingreifen, Satan und seine Höllenscharen sich hingegen als Kriegstreiber betätigen. Geboten werden ganz epos-typisch groß angelegte Reden Gottes und Satans sowie dann auf der Ebene menschlicher Akteure Torstensons, der seinen Soldaten Mut zuspricht, und kaiserlicher Soldaten, die gottlose Reden schwingen. ${ }^{26}$ An einigen Stellen erreicht der Text darüber hinaus dramatische Dimensionen, etwa wenn ein veritabler Dialog zwischen Akteuren geboten wird. ${ }^{27}$ Auffällig ist, dass demgegenüber die Schlacht selbst gemessen am Umfang des Texts nicht sehr ausführlich und detailliert geschildert wird. ${ }^{28}$ Sehr viel Aufmerksamkeit wird hingegen der Beschreibung des Schlachtfelds nach der Schlacht gewidmet.

An einigen Stellen des Texts macht Illenhöfer auf ein Problem aufmerksam: Es ist nicht leicht, angesichts der Konstellation vor Breitenfeld eine klare Freund-Feind-Unterscheidung zu treffen, wenn man ein redlicher, protestantischer Deutscher in schwedischen Diensten ist. Einerseits ist klar, dass die Schweden der protestantischen und damit aus Sicht des Autors gerechten Sache dienen - einen vor den Schweden fliehenden kaiserlichen Reiter lässt Illenhöfer ausrufen, »GOtt in dem Himmel selbst « sei wohl Schwedisch geworden $^{29}$-, andererseits ist die deutsche Seite am ehesten jene der Kaiserlichen, unter denen sich auch viele deutsche Protestanten finden. Diese Seite wird bei der Beschreibung des Schlachtfeldes nach Ende der Schlacht durch einen ausführlichen »Trawerblick « des Dichters gewürdigt, ${ }^{30}$ der sich zu einer seitenlangen evidentia-Figur auswächst, einem rhetorischen Voraugenführen des Schreckens, der sich auf dem Schlachtfeld dem Betrachter darbietet. Eingeleitet wird diese häufende Beschreibung mit dem Gedanken, dass auf diesem Schlachtfeld tausende Tote beisammen liegen, die sich gegenseitig umgebracht haben, obwohl sie einander überhaupt nicht gehasst haben, und von denen die allermeisten, könnte man sie fragen, wohl nicht in der Lage sein

26 Illenhöfer: Poetische Beschreibung (s. Anm. 1), Bl. B $4^{\mathrm{v}}$ f., V. 385-420 [Rede Gottes]; Bl. C $1^{\mathrm{r}}-$ C 2 ${ }^{\mathrm{r}}$, V. 427-481 [Rede Satans]; B. C $2^{\mathrm{r}}$ f., V. 500-526 [Rede Torstensons].

27 Ebd., Bl. D $3^{\mathrm{v}}$ f., V. 889-931. Ich zitiere den Beginn des Dialogs, der in der Marginalie als »Des Commendanten zu Wittenberg vnd d flüchtigen Käys: Reuter Gespräch« ausgewiesen ist: »Erbärmlich flehen sie: Reuter. O nehmt vns ärmsten ein/ | Daß wir vorm Schwerdte nur des Feindes sicher seyn. | Commend: Was Volckes seyd jhr denn? das mit so tollen Sitten | Jhr Wahlfahrten hierher zu Luthern kommt geritten? | Reuter. O viva Ferdinand! Herr Luther kan dißmal | Erretten/ so jhr vns einlasst/ ein hohe Zahl.« Und so weiter.

28 Ebd., Bl. C $4^{\mathrm{v}}-\mathrm{D} 2^{\mathrm{r}}$, V. 674-777.

29 Ebd., Bl. D $4^{\mathrm{r}}$, V. $924 \mathrm{f}$.

30 Ebd., Bl. E 3 ${ }^{\mathrm{r}}$, V. 1143-1145: „O welch ein Trawerblick erfüllet mein Gesichte! | Seynd alles Christen? Ja. Seynd das der Liebe Früchte? | Ja. Wie sie heute gehn.«. 
würden zu sagen, aus welchem Grund sie hier sterben mussten. ${ }^{31}$ Es folgen Beschreibungen der Leichen auf dem Schlachtfeld und ihrer Versehrungen, die aufgrund ihrer Drastik für heutige Leser einen Realitätseffekt zu markieren scheinen, die aber in Schlachtbeschreibungen der Zeit durchaus topisch auftauchen: abgerissene Körperteile, aus dem Leib tretende Innereien und Ähnliches. ${ }^{32}$

Auf eine Passage, in der die Schrecken des Schlachtfeldes in dieser Weise konkret beschrieben werden, folgt eine andere, in der der Dichter als Kriegsberichterstatter selbst als Figur auf dem Schlachtfeld erscheint, Interviews mit den Sterbenden führt und sie insbesondere nach ihrer Herkunft fragt. ${ }^{33}$ Diese Passage entwickelt sich zu einem epischen Katalog, der evident macht, dass die auf dem Schlachtfeld gefallenen Kaiserlichen von allen Orten Deutschlands und darüber hinaus stammen. Hier findet eine Inversion einer Vorgabe der Gattungstradition statt: Listen Kataloge seit Homer typischer Weise die Truppen auf, die zur Schlacht aufmarschieren, katalogisiert Illenhöfer die Gefallenen nach der Schlacht, betont also statt der Staunen erregenden Kriegsmacht die Betroffenheit auslösenden Verluste. 47 Personen und ihre konkreten Herkunftsorte von Meißen über Mainz, Passau und Stettin bis Buxtehude werden benannt, ${ }^{34}$ bis der Dichter mit einem Unsagbarkeitstopos schließt: »Unmöglich wars zuletzt/ zu mercken Stadt vnd Land/ | Daraus die Pursche sich/ so lebte

31 Ebd., Bl. E 3v', V. 1151-1156: »Hier ligen ohne Zahl viel Tausend todgeschlagen/ | Die sonst zusammen nie gar keinen Haß getragen/ | Ja wohl jhr lebenlang einander nichts gethan/ I Und wenn der zwanzigste solt jetzo zeigen an/ | Warumb er diesen Tod vor dißmal leiden müssen/ | Er wird ein Ursach kaum dißfalls zu sagen wissen.".

32 Ebd., Bl. E $4^{\mathrm{r}}$, V. $1187 \mathrm{f}$.: »Bey jenem eine Faust vom Arme abgeschnitten/ | Gelt! Wo der künfftliglich wird auf der Trommel wüten.« Ebd., V. 1217-1219: »Der lieget ohne Kopff/ der ohn die rechte Hand/ | Dem ist die lincke gantz vom Leibe weggebrannt | Durch fewriges Geschoß.« Ebd., Bl. E 4v, V. 1223: „Dem ist der Leib zerschnitten/ | Daß auf die Erden er die Därme müssen schütten.« Vgl. zur Topik drastischer Beschreibungen in diesem Kontext Dirk Werle: Erzählen vom Dreißigjährigen Krieg. Hannover 2020.

33 In der Randnote ausgewiesen als »Des Authoris Gespräche mit etlichen verwundet Käyserl. Auf der Wahlstatt«, Illenhöfer: Poetische Beschreibung (s. Anm. 1), Bl. F $1^{\mathrm{r}}$.

34 Punktuell unter Nachahmung der entsprechenden Dialekte der jeweiligen Landsleute: »Der zwey vnd vierzigste war aus dem Oberland/ I Und zwar von Woiblinga/ da Schwvaba wuol bekannt.« Ebd., Bl. F 2 ${ }^{\mathrm{r}}$, V. 1339 f. Vgl. auch die Nachahmung des Akzents eines bei den Kaiserlichen mitkämpfenden Franzosen: »Kein Teutsche/ sagten wir/ ist das/ das ist gewiß/ | Da sprach er böse Teutsch: Bin ich von der Paris, | VVo vvohn der gut Frantzos, du liebe Teutsche Bruder." Ebd., Bl. F 1 ${ }^{\text {v }}$, V. 1325-1327. Vgl. für ein anderes frühneuzeitliches Beispiel der Imitation von >Franzosen-Deutsch` Kai Bremer u. a.: Formen der Geselligkeit und ihr historischer Wandel als Herausforderung der frühneuzeitlichen Kulturgeschichte. Das Beispiel Leipzig. In: Daphnis 49 (2021), S. 1-13. 
noch/ genannt «. ${ }^{35}$ Unterfüttert wird die empathische Schilderung der gefallenen und sterbenden Kaiserlichen auf dem Schlachtfeld durch eindrückliche Details, etwa jenes des Soldaten, der den Dichter und Kriegsberichterstatter bittet, seinem alten Vater, zu dem er als sein einziger Sohn nicht mehr zurückkehren wird, von seinem ehrenvollen Tod zu berichten, oder jener tödlich Verwundeten, die vor Schmerzen versuchen, sich mit ihren Fingernägeln ins Erdreich hineinzuwühlen. ${ }^{36}$

Mit einem harten Bruch geht der Dichter von dieser beteiligten Schilderung des sinnlosen, massenhaften Sterbens seiner Landsleute über zum - frühneuzeitliche carmina heroica häufig als Strukturmerkmal bestimmenden - Rühmen des Helden, des schwedischen Feldherrn Torstenson: „Genug von dieser Schlacht Jetzt wend ich mich zu Dir | Du freyer Norden-Held «. ${ }^{37}$ Es folgt die ausführliche, den Gerühmten über weite Strecken apostrophierende Epideixis Torstensons, die sich nicht zuletzt in der Einschätzung ausspricht: „Dein Ruhm ist mehr/ daß du sehr vielen hast das Leben | Erhalten/ als geraubt ${ }^{38}$ - eine bemerkenswerte Aussage nach der ausführlichen Darstellung Sterbender auf dem Schlachtfeld. Torstenson wird vom Dichter darüber hinaus als legitimer Nachfolger Gustav Adolfs präsentiert, was jenem Gelegenheit gibt, in einer gleichfalls epos-typischen umfangreichen, in diesem Fall mehrere hundert Verse umfassenden Analepse die Vorgeschichte der zweiten Schlacht von Breitenfeld von Gustav Adolfs Invasion 1631 an über die Verheerung Magdeburgs durch Tillys Truppen und die erste Schlacht von Breitenfeld im selben Jahr, den Tod Gustav Adolfs in der Schlacht von Lützen 1632 und darüber hinaus zu rekapitulieren. ${ }^{39}$ Das carmen heroicum über die zweite Schlacht von Breitenfeld endet mit der Evokation eines Triumphzugs, in dem der Kriegsheld Torstenson auf einem goldenen Wagen auf den Tempel der Fama zufährt. ${ }^{40}$

Mit dem bis hierher Gesagten ist die Eigenart von Illenhöfers Text bei weitem nicht erschöpfend erfasst, gleichwohl sei hier die Vorstellung und Analyse

35 Illenhöfer: Poetische Beschreibung (s. Anm. 1), Bl. F 2 ${ }^{\mathrm{r}}$, V. 1359 f.

36 Ebd., Bl. F 1, V. 1298-1305: »[...] der zwölffte von Cüstrin/ | Und vor ein eintzig Kind des Pfarrers sich erklärte/ | Derselbe bis an vns wehmütiglich begehrte/ | Daß/ so je einer einst allda durchreisen solt/ | An seinen Vater man nur dieses bringen wolt/ | Er sey ins Käysers Dienst vmb Leib vnd Leben kommen/ | Wiewol man listig jhn vnd Schelmisch weggenommen | In offnem Kruge hett.« Ebd., Bl. F 2 v, V. 1365-1367: »Man sahe/ wie von vielen | Vor Pein versuchet ward sich in die Erd zu wühlen | Durch jhrer Nägel Krafft/ eh als die Seel entfahrn [...].«

37 Ebd., Bl. F 2v , V. $1399 \mathrm{f}$.

38 Ebd., Bl. F 3 $3^{\mathrm{v}}$, V. $1450 \mathrm{f}$.

39 Ebd., Bl. G $1^{\mathrm{r}}-\mathrm{I} 1^{\mathrm{r}}$, V. 1551-3056 [recte: 2156].

40 Ebd., Bl. I 1 , V. 3082-3084 [recte: 2182-2184]. 
des Texts abgebrochen, da ja im Rahmen dieses Bandes danach zu fragen ist, was man aus diesem Fallbeispiel mit Blick auf ideen- und sozialgeschichtliche Perspektiven der deutschen Literaturwissenschaft nach dem Ende des cultural turn ablesen kann. Dieser Frage gehe ich umso dankbarer nach, als die Beschäftigung mit ihr methodologische Klärung über Fragen verspricht, mit denen meine Mitarbeiter^innen und ich uns seit einiger Zeit in dem von der DFG geförderten Forschungsprojekt »Epische Versdichtungen des >langen derts (1570-1740) im deutschen Kulturraum« beschäftigen. Im Rahmen dieses Projekts erarbeiten wir ein literaturhistorisches Repertorium, in dem wir ein Korpus von etwa 120 einschlägigen Texten literaturhistorisch erschließen und vorstellen, darunter selbstverständlich auch Illenhöfers Poetische Beschreibung der Schlacht von Breitenfeld.

\section{Methodologische Implikationen und Konsequenzen}

Die Herausgeber des vorliegenden Bandes konfrontieren ihre Autor*innen und die geneigten Leser ${ }^{\star}$ innen mit einem theoriegeschichtlichen Narrativ, das aus zwei Teilen besteht. Teil 1: Es hat in der Literaturwissenschaft der letzten Jahre ein kulturwissenschaftliches Paradigma gegeben, und dieses Paradigma hat sich nunmehr erschöpft. Teil 2: Nun ist der Weg frei für etwas Neues, und dieses Neue ist etwas Alt-Neues: Anzuknüpfen ist an eine Ideengeschichte der Literatur einerseits, an eine Sozialgeschichte der Literatur andererseits, und gerade in der produktiven Interaktion, vielleicht sogar hervorgehend aus Debatten und Kontroversen, soll eine angemessene und zeitgemäße Form von Literaturwissenschaft und Literaturgeschichte sichtbar werden. Zu diesem Zweck haben die Herausgeber je zwei Expert`innen für die deutsche Literaturwissenschaft jedes Jahrhunderts vom 12. bis zum 21. eingeladen, von denen je einer, je einem hier die Rolle der Ideenhistorikerin, des Ideenhistorikers, dem bzw. der anderen die Rolle der Sozialhistorikerin, des Sozialhistorikers zufällt. Das so etwas grob skizzierte Narrativ besitzt eine Reihe von Unschärfen. Kann es zum Beispiel sein, dass es die Kulturwissenschaft als homogenen Komplex eigentlich nie gegeben hat? Kann es außerdem sein, dass gerade die zur Beschreibung der Lage in den 1990er und 2000er Jahren häufig kolportierte Frontenbildung >Kulturwissenschaft vs. Rephilologisierung mehr ein politisch-strategisches Vereinfachungsnarrativ ist, als dass es eine literaturwissenschaftliche Wirklichkeit abbildete? Und ist es plausibel, dass gerade die Verknüpfung von Sozial- und Ideengeschichte Innovationspotential in Methodenfragen besitzen sollte? 
Warum sollte das so sein? Als Sozialgeschichte der Literatur ging das dominante literaturwissenschaftliche Theorieangebot der 1970er und 1980er Jahre in die Fachgeschichte ein; warum sollte man ausgerechnet das jetzt wieder aufgreifen? Von einer >Ideengeschichte der Literatur/ hingegen hat als nennenswerter Strömung bislang niemand gesprochen; ein entsprechendes Lemma im Reallexikon oder in einem vergleichbaren Wörterbuch habe ich jedenfalls nicht gefunden.

Trotz dieser Unschärfen kommt mir das Narrativ zum vorliegenden Band triftig vor. Mein Vorschlag wäre aber, es nicht im Sinne eines programmatischen Theorie- und Methodenwechsels zu interpretieren - Ideen- und Sozialgeschichte gemeinsam gegen die Kulturwissenschaft und gleichzeitig auch gegeneinander sondern als Einladung, über einige grundlegende Fragen und Probleme der Literaturgeschichtsschreibung nachzudenken. Eines haben die hinter den Labels Kulturwissenschaft, Sozialgeschichte und Ideengeschichte sich möglicherweise versteckenden Programme in dieser Hinsicht schon einmal gemeinsam; soviel kann man sagen, ohne die drei Konzepte genauer und ausführlicher explizieren zu müssen. Alle drei stehen nicht zuletzt für Versuche, eine anspruchsvolle Form von Literaturgeschichte zu konzipieren, die über die reine Prosopographie, das Aufzählen von Namen, Daten und Fakten hinausgeht. Ein solches prosopographisches Unterfangen gilt heute in weiten Bereichen der Literaturwissenschaft als gestrig und unangemessen, aber in weiten anderen Bereichen wird es nach wie vor mit Hingabe gepflegt. Mit einem rein prosopographischen Zugriff kommt man nun beispielsweise, wenn man auf das Fallbeispiel Illenhöfer blickt, nicht sehr weit. So ist etwa über den Autor des Texts, aber auch über die Offizin, in der der Text verlegt und gedruckt wurde, und über viele weitere harte Fakten nichts herauszubekommen. Man muss hier mit anderen, differenzierteren und subtileren Mitteln vorgehen. Wenn man sich in diesem Kontext fragt, warum Illenhöfers Poetische Beschreibung der Schlacht von Breitenfeld bislang nicht durch eine literaturwissenschaftliche Untersuchung erschlossen ist, dann hat ein Teil der Antwort mit dem Charakter dieses Texts und seiner Überlieferung zu tun: Wir wissen nichts über den Autor, und der Text ist ein Lobgedicht auf einen schwedischen Heerführer. Die ältere Germanistik konnte mit einem solchen Text nichts anfangen, weil zwei ihrer dominanten Interessen, das Verhältnis von Texten zu ihren Autoren und die kulturelle Vorläuferrolle der deutschen Literatur für die Etablierung eines deutschen Nationalstaats, von diesem Text nicht berührt wurden. In der jüngeren Germanistik hingegen hat sich kaum noch jemand für epische Versdichtungen interessiert, weil man sich bei der Erforschung von Erzähltexten und ihrer Geschichte auf die moderne Leitgattung des Romans konzentrierte.

Illenhöfers epische Versdichtung liegt nicht in einer modernen Edition vor und ist nicht weit verbreitet, nur in den Universitäts- und Landesbibliotheken 
in Halle und Dresden haben sich Exemplare des Drucks erhalten. Dass es im Jahr 2022 gleichwohl leicht möglich ist, den Text als Fallbeispiel für eine Reflexion auf allgemeinere methodologische Fragestellungen heranzuziehen, hat mit einer Entwicklung zu tun, die sich unabhängig von Fragen der Kulturwissenschaft, Sozial- und Ideengeschichte abspielt: Der Text ist als Digitalisat der Universitäts- und Landesbibliothek Sachsen-Anhalt im Internet zugänglich; er ist damit Teil einer medientechnischen Entwicklung, die die Frühneuzeitforschung in den letzten Jahren eminent verändert hat. Eng verknüpft mit diesem medientechnischen Fortschritt ist die rasante Entwicklung eines Forschungsfeldes und eines damit verbundenen wissenschaftlichen Zugriffs auf Literatur, die mit dem Label Digital Humanities noch recht global und unscharf bezeichnet sind. Diesen Siegeszug kann man, wenn man sich Anfang der 20er Jahre des 21. Jahrhunderts mit methodologischen Fragen der Literaturgeschichte beschäftigt, eigentlich nicht außer Acht lassen; man müsste sich etwa fragen, in welchem Verhältnis ein close reading, wie es im ersten Teil dieses Beitrags ansatzweise vorgeführt wurde, mit Formen des distant reading ${ }^{41}$ steht, stehen kann, stehen sollte, die durch technische Fortschritte im Bereich der Digital Humanities zunehmend ermöglicht werden. Das kann an dieser Stelle nicht weiter vertieft werden, denn es soll ja um das Verhältnis von Kultur- und Sozialgeschichte gehen.

Der cultural turn, der mit dem Titel des vorliegenden Bandes als überwunden dargestellt wird, hat, wenn man das überhaupt so großflächig sagen kann, für die germanistische Literaturwissenschaft Transformationen bewirkt, die dem Fach gutgetan haben. $\mathrm{Zu}$ nennen sind hier etwa die theoretische Fundierung einer Erweiterung des Literaturbegriffs, die Etablierung von Forschungsperspektiven auf die Materialität der Literatur und das kulturelle Gedächtnis. Wenn in manchen Bereichen der Literaturwissenschaft über einen längeren Zeitraum hinweg etwas perhorresziert wurde, das man >Kulturwissenschaft « nannte, dann meinte man damit vorrangig nicht etwa die genannten Tendenzen oder gar Versuche, kulturgeschichtliche Kontexte bei der Interpretation literarischer Texte besonders stark zu berücksichtigen, sondern man meinte andere Tendenzen, die man zu beobachten glaubte und die auf eine Ersetzung der wissenschaftlichen Leitdifferenz >wahr - falsch « durch die ästhetische Leitdifferenz sinteressant - uninteressant ` abzuzielen schienen. ${ }^{42}$

41 Vgl. dazu programmatisch Franco Moretti: Distant Reading. Aus dem Englischen übersetzt von Christine Pries. Konstanz 2016 [Orig. London 2013].

42 Vgl. Dirk Werle: Jenseits von Konsens und Dissens? Das Interessante als kulturwissenschaftliche Beschreibungskategorie. In: Internationales Archiv für Sozialgeschichte der Literatur 30 (2005), S. 117-135. 
Wie stark diese Tendenzen wirklich gewesen sind, müssen vielleicht in einigen Jahren Wissenschaftsforscher ${ }^{\star}$ innen $\mathrm{zu}$ rekonstruieren versuchen. ${ }^{43}$ Jedenfalls aber glaubt, so unscharf das Label Kulturwissenschaft auch ist, jede und jeder, die oder der Teil der community ist, irgendwie und intuitiv zu wissen, was damit gemeint ist.

Und wenn man sich durch die Brille dieses Labellings einmal die durchaus überschaubare Forschungssituation zum carmen heroicum in der frühen Neuzeit ansieht, dann kann man eine interessante Beobachtung machen: Genuin sich als kulturwissenschaftlich verstehende Beiträge zu diesem Forschungsfeld fehlen fast ganz; eine Ausnahme bildet vielleicht ein Beitrag von 2015, der in der Tat der Befürchtung, >die Kulturwissenschaftı ersetze die Leitdifferenz `wahr - falsch ` durch jene sinteressant - uninteressant`, ein wenig Vorschub leistet, denn in dem Beitrag wird eine interessant klingende These tendenziell um den Preis historischer Korrektheit vorangetrieben. In dem besagten Beitrag wird für die These argumentiert, im Bereich der Gattung carmen heroicum habe im 17. Jahrhundert eine schrittweise Ablösung des Heldengedichts durch das Lehrgedicht stattgefunden, und damit habe sich die Art, wie im literarischen Text Wissen konstituiert werde, grundlegend verändert. ${ }^{44}$ Diese These klingt gut, aber sie wird durch die historische Materiallage allenfalls rudimentär gestützt. Helden- und Lehrepik bestehen durch das 17. Jahrhundert hindurch und bis ins 18. hinein nebeneinander fort und gehen nicht selten vitale Interaktionen ein, wie ja auch etwa in Illenhöfers Poetischer Beschreibung der Schlacht von Breitenfeld, die Elemente eines Heldengedichts und einer Lehrdichtung, oder besser: eines journalistischen Berichts, in eine interessante Spannung bringt.

Keinen systematischen Ort findet die Gattung des carmen heroicum in dem 2013 erschienenen interdisziplinären Handbuch Literatur und Wissen, obwohl es sich doch, wie angedeutet, um die literarische Gattung handelt, in der die Vermittlung von Wissen durch Dichtung als genrekonstitutiv gesehen wurde. Allein in einer fünfseitigen exemplarischen Lektüre von Opitz' 1631 veröffentlichtem Lehrepos Vesuvius gibt Jörg Robert einen - freilich sehr hellsichtigen -

43 Vgl. für erste Explorationen in diesem Feld Serjoscha P. Ostermeyer: Der Kampf um die Kulturwissenschaft. Konstitution eines Lehr- und Forschungsfeldes - 1990-2010. Berlin 2016.

44 Andreas B. Kilcher: >Litteraturく. Formen und Funktionen der Wissenskonstitution in der Literatur der Frühen Neuzeit. In: Frank Grunert, Anette Syndikus (Hg.): Wissensspeicher der Frühen Neuzeit. Formen und Funktionen. Berlin, Boston 2015, S. 357-375. Vgl. ebd., S. 364: »Wenn dem Epos auch nach dem 17. Jahrhundert eine enzyklopädische Funktion zugesprochen werden konnte, dann galt dies [...] nicht mehr so sehr für das sheroisch getichte (Opitz), d. h. für das Heldenepos im homerischen Sinn. Dieses wurde in der Funktion mehrheitlich durch das `carmen didacticum`, das Lehrgedicht, abgelöst. Analog zum Heldenepos kann man geradezu von einem `enzyklopädischen Lehrgedicht` sprechen.«. 
Einblick in die wissenshistorische Relevanz des Genres, aber dieser Absatz bleibt als vereinzeltes Fallbeispiel, das im Rest des Handbuchs keine Resonanz findet, konzeptionell eher randständig. ${ }^{45}$ Das kann man freilich nicht dem Verfasser zur Last legen, im Gegenteil, es ist ihm hoch anzurechnen, dass er die wichtige Thematik für das Handbuch gerettet hat; es liegt anscheinend an einer mangelnden Sensibilität der Herausgeber`innen für die Wichtigkeit des Genres carmen heroicum mit Blick auf das im Handbuch erschlossene Themengebiet. Und diese mangelnde Sensibilität hat wiederum vielleicht etwas mit dem Umstand zu tun, dass die Herausgeber*innen des Handbuchs Literatur und Wissen einer Unterströmung der kulturwissenschaftlichen Literaturwissenschaft zuzurechnen sind, der >Poetologie des Wissens`. Diese kulturwissenschaftliche Spielart der Wissenschafts- und Wissensgeschichte geht davon aus, dass literarische Formen gelehrtes und wissenschaftliches Wissen konstitutiv hervorbringen, ${ }^{46}$ wogegen konservativere Konzeptionen der Wissenschafts- und Wissensgeschichte erst einmal davon ausgehen würden, dass literarische Formen etwas den Wissensgehalten grundsätzlich Nachgängiges sind und dass Literatur auf Wissenschaft reagiert, nicht aber die frühere und bessere Wissenschaft ist. ${ }^{47}$ Mit Blick auf die Gattungsgeschichte des carmen heroicum muss man sagen, dass man mit einer >Poetologie des Wissens` hier nicht sehr weit kommt. Die großenteils eher sperrigen Texte widersetzen sich einem derartigen Zugriff und sind für ihn damit uninteressant, sie fordern einen traditionelleren, dabei gleichwohl differenzierten wissenshistorischen Zugang.

Die Opposition >Ideengeschichte versus Sozialgeschichte`, in deren Spannungsfeld gemäß dem - vorliegendem Band zugrunde liegenden - Narrativ >nach der Kulturgeschichte` der angemessene literaturwissenschaftliche Zugriff auf Texte und ihre Geschichte erfolgen soll, ist auf den ersten Blick eine

45 Vgl. Jörg Robert: Martin Opitz: Vesuvius Poëma Germanicum (1633). In: Roland Borgards u. a. (Hg.): Literatur und Wissen. Ein interdisziplinäres Handbuch. Stuttgart 2013, S. 301-305. Von dieser Fehlanzeige lässt auch Wilhelm Kühlmann seinen monographischen Überblick zur frühneuzeitlichen Lehrdichtung starten: Wilhelm Kühlmann: Wissen als Poesie. Ein Grundriss zu Formen und Funktionen der frühneuzeitlichen Lehrdichtung im deutschen Kulturraum des 16. und 17. Jahrhunderts. Berlin, Boston 2016, S. 1, Anm. 1.

46 Vgl. dazu handbuchartig-kodifizierend Armin Schäfer: Poetologie des Wissens. In: Roland Borgards u. a. (Hg.): Literatur und Wissen. Ein interdisziplinäres Handbuch. Stuttgart 2013, S. 36-41.

47 Vgl. Lutz Danneberg, Carlos Spoerhase: Wissen in Literatur als Herausforderung einer Pragmatik von Wissenszuschreibungen: sechs Problemfelder, sechs Fragen und zwölf Thesen. In: Tilmann Köppe (Hg.): Literatur und Wissen. Theoretisch-methodische Zugänge. Berlin, New York 2011, S. 29-76; Andrea Albrecht u. a.: Zum Konzept Historischer Epistemologie. In: Scientia Poetica 20 (2016), S. 137-165. 
überraschende Setzung, denn erstens spricht heute aus hier nicht genauer zu erörternden Gründen kaum noch jemand von Ideengeschichte, sondern eher in einem erweiternden Sinne von intellectual history, und ebenso spricht kaum noch jemand von Sozialgeschichte, sondern eher in einem erweiternden Sinne von Gesellschaftsgeschichte. Und zweitens ist die Trennung von Gesellschaftsgeschichte und intellectual history sinnvoller Weise überhaupt nicht säuberlich vorzunehmen: Ideen sind ja stets in ihren gesellschaftlichen Kontexten eingebettet, und Gesellschaft ist nicht anders zu verstehen denn über ihre intellektuellen Hintergründe. Die Opposition Ideen- versus Sozialgeschichte scheint jedoch ebenfalls für eine grundsätzliche Frage der Textinterpretation und der Literaturgeschichtsschreibung zu stehen: Welche Kontexte sind beim historischen und interpretierenden Umgang mit Texten zu favorisieren: intertextuelle das wäre die ideengeschichtliche Option - oder extratextuelle - das wäre die sozialgeschichtliche Option? Die Frage lässt sich allerdings so nicht beantworten. $\mathrm{Zu}$ ersetzen ist sie, wie ich mir einzubilden nicht abgewöhnen kann, durch eine problemgeschichtliche Perspektive und damit durch die Frage: Was könnte die Problemsituation sein, auf die der vorliegende Text oder die vorliegende Textgruppe reagiert? ${ }^{48}$ Je nachdem kann diese Problemsituation eher gesellschaftlicher oder eher ideenhistorischer Natur sein, zumeist wird aber beides eine Rolle spielen. ${ }^{49}$ Am Beispiel Illenhöfers: Für das Verständnis des Texts sind nicht die gesellschaftlichen Kontexte Krieg und Gefolgschaft und nicht die ideengeschichtlichen Kontexte Heldenlob und Klageritual vorrangig, auch nicht der Kontext Nationalitätsbewusstsein, von dem es gar nicht leicht ist zu sagen, ob er eher gesellschaftlicher oder eher ideengeschichtlicher Natur ist. Nein, alle diese Kontexte spielen eine Rolle hinsichtlich der zentralen im Text reflektierten und thematisierten, keineswegs gelösten, sondern in der Spannung gehaltenen Problemsituation, die sich durch die Frage umreißen lässt: Welche Position nehme ich als protestantischer Deutscher in einem kriegerischen Konflikt ein, in dem die Kaiserlichen am ehesten Deutschland und die deutsche Sache

48 Vgl. zum Konzept literaturwissenschaftlicher Problemgeschichte Dirk Werle: Modelle einer literaturwissenschaftlichen Problemgeschichte. In: Jahrbuch der Deutschen Schillergesellschaft 50 (2006), S. 478-498; ders.: Frage und Antwort, Problem und Lösung. Zweigliedrige Rekonstruktionskonzepte literaturwissenschaftlicher Ideenhistoriographie. In: Scientia Poetica 13 (2009), S. 255-303, und die durch diesen Beitrag eingeleitete Forschungsdiskussion in den Bänden 13 (2009) und 14 (2010) des Jahrbuchs Scientia Poetica sowie ders.: Problem und Kontext. Zur Methodologie der literaturwissenschaftlichen Problemgeschichte. In: Journal of Literary Theory 8 (2014), S. 31-54.

49 Vgl. in diesem Sinne bereits Karl Eibl: Literaturgeschichte, Ideengeschichte, Gesellschaftsgeschichte - und `Das Warum der Entwicklung`. In: Internationales Archiv für Sozialgeschichte der deutschen Literatur 21 (1996), S. 1-26. 
repräsentieren, der schwedische Heerführer hingegen als protestantischer Heros agiert und firmiert?

Wer sich für literaturhistorische Verlaufsformen interessiert, für den literarischen Wandel, seine Dynamiken und Bedingungen, der muss sich von den literaturwissenschaftlichen Basisbeschreibungskonzepten vor allem für das Konzept der Gattung interessieren. Autor^innen und ihre Werke sind ziemlich individuelle Einheiten, an denen man keinen Wandel ablesen kann; Epochenbegriffe implizieren die Vorstellung ziemlich statischer Makroformationen, die differenziertere Einsichten über literarischen Wandel und literaturhistorische Verläufe eher behindern als befördern. Gattungen hingegen sind historisch entwickelte Formen, innerhalb derer sich literarische Produktion und Rezeption wesentlich abspielt und eine geschichtliche Dynamik entfaltet. Auch Gattungsgeschichte lässt sich jedoch angemessen nur im Miteinander von sozial- und ideengeschichtlicher Perspektive rekonstruieren. Gattungen sind einerseits, mit Wilhelm Voßkamp gesprochen, »>Bedürfnissynthesen ‘ [...], in denen nicht nur bestimmte Problemlagen artikuliert, sondern auch Lösungsstrategien diskutiert und angeboten werden, die Möglichkeiten (zeitlich begrenzter) Bedürfnisbefriedigung für bestimmte Leser (Schichten, Gruppen) bieten. ${ }^{50}$ Sie lassen sich mithin als Reaktionen auf gesellschaftliche Bedürfnisse verstehen. Andererseits entwickeln Gattungen im Wechselspiel von Gattungstradition, Gattungsbewusstsein und individuellem Reagieren auf Gattungserwartungen, von Tradition und Traditionsverhalten, eine interne Dynamik mit einer Eigenlogik. ${ }^{51}$ Gattungen sind so gesehen Ideen, Gattungsgeschichte ist eine Form von Ideengeschichte. Eine anspruchsvolle Gattungsgeschichte muss beide Aspekte, den sozialgeschichtlichen und den ideengeschichtlichen, berücksichtigen, und das lässt sich in den meisten Fällen auch gar nicht vermeiden. So zeigt sich etwa in der Lektüre von Illenhöfers Poetischer Beschreibung der Schlacht von Breitenfeld, wie der Autor die verschiedenen Möglichkeiten, die die Gattung des carmen heroicum bereit-

50 Wilhelm Voßkamp: Thomas Morus' Utopia: Zur Konstituierung eines gattungsgeschichtlichen Prototyps. In: Ders. (Hg.): Utopieforschung. Interdisziplinäre Studien zur neuzeitlichen Utopie. Bd. 2. Frankfurt a.M. 1985 [zuerst 1982], S. 183-196, hier S. 183.

$51 \mathrm{Vgl}$. zum in diesem Zusammenhang weiterführenden Konzept des literarischen Traditionsverhaltens Wilfried Barner: Wirkungsgeschichte und Tradition. Ein Beitrag zur Methodologie der Rezeptionsforschung [1975]. In: Ders.: Pioniere, Schulen, Pluralismus. Studien zu Geschichte und Theorie der Literaturwissenschaft. Tübingen 1997, S. 253-276; ders.: Über das Negieren von Tradition. Zur Typologie literaturprogrammatischer Epochenwenden in Deutschland. In: Reinhart Herzog, Reinhart Koselleck (Hg.): Epochenschwelle und Epochenbewusstsein. München 1987, S. 3-51; ders.: Tradition als Kategorie der Literaturgeschichtsschreibung [1988]. In: Ders.: Pioniere, Schulen, Pluralismus. Studien zu Geschichte und Theorie der Literaturwissenschaft. Tübingen 1997, S. 277-296. 
hält, nutzt, um literarisch auf die geschilderte Problemsituation zu reagieren und sie im epischen Format zu reflektieren: Schlachtbeschreibung, Heldenlob, Nationenkatalog, Musenanruf, ${ }^{22}$ Artikulation eines dichterischen Wahrheitsanspruchs.

Ideenhistorische und sozialhistorische Perspektive haben noch in einer anderen Hinsicht eine Gemeinsamkeit: Sie implizieren eine Abkehr von einer in der Literaturwissenschaft immer noch verbreiteten Konzentration großer Anteile der Forschungskapazitäten auf kanonische, als ästhetisch besonders wertvoll erachtete Texte. Eine anspruchsvolle Ideengeschichte der Literatur kann nur schreiben, wer nicht nur die kanonischen Texte von Wolfram von Eschenbach, Goethe, Schiller und Thomas Mann im Blick hat, sondern vor allem auch die vielen wenig bekannten Texte dazwischen, die es erlauben, eine historische intellektuelle Dynamik, wie sie sich in Texten niederschlägt, überhaupt erst erkennbar werden zu lassen. Und wer sich für sozialgeschichtliche Dimensionen der Literatur interessiert, wird - wenn man einmal exemplarisch auf die Erzählliteratur des 17. Jahrhunderts schaut - vielleicht in einem zeitgenössischen Bestseller wie Johann Michael Moscheroschs Wunderbarlichen und wahrhafftigen Gesichten Philanders von Sittewalt mehr relevante Funde machen können als in einem nachträglich hochkanonisierten, aber in seiner Zeit weniger erfolgreichen Text wie Hans Jacob Christoffel von Grimmelshausens Simplicissimus Teutsch. Entsprechend ist ein Text wie die Poetische Beschreibung Illenhöfers im Kontext einer kanonorientierten Literaturgeschichte überhaupt nicht sinnvoll thematisierbar; es handelt sich vor diesem Hintergrund um einen belanglosen, irrelevanten, vernachlässigenswerten Text. Eine solche Literaturgeschichte würde die Geschichte deutschsprachiger epischer Versdichtungen vielleicht überhaupt erst mit Friedrich Gottlieb Klopstocks Messias anfangen lassen; die zahlreichen einschlägigen Texte, die innerhalb dieser Genretradition seit dem 16. Jahrhundert entstanden sind, würden durch den kanonorientierten Radar einfach nicht erfasst werden. Im Kontext einer ideen- und sozialhistorisch informierten Gattungsgeschichte hingegen wird deutlich, wie ein scheinbar randständiger Text wie der Illenhöfers eine beträchtliche literaturhistorische Signifikanz entfalten kann.

Die hier skizzierten Überlegungen zum notwendigen Miteinander von Kontextualisierungen intertextueller und extratextueller Natur sowie der Perspektive auf literarische Gattungen sowohl als gesellschaftliche Bedürfnissynthesen als auch als Traditionen mit Eigenlogik und schließlich zum Verhältnis

52 In diesem Fall modifiziert zu einem Anruf Apollos, mit dem der Text einsetzt: »APollo, schenck mir nun was reicher deinen Safft/ | Daß meine Feder sich erheb in dessen Krafft/ | Zu fliegen in das Lob viel edler Krieges-Helden; | Die güldne Leyer stimm; komm/ komm/ hilff mir vermelden | Die ritterliche Schlacht [...].« Illenhöfer: Poetische Beschreibung (s. Anm. 1), Bl. A 3v , V. 1-5. 
von Literaturgeschichte und literarischem Kanon lassen sich nochmals in einer spezifisch wissenschaftshistorischen Weise perspektivieren, die die entwickelten Sachverhalte zusammenzufassen und etwas anders zu konturieren erlaubt: Die literaturwissenschaftliche Sozialgeschichte seit den 1970er Jahren entdeckte schnell als eines ihrer liebsten Spielfelder die Barockforschung. Und das geschah nicht ohne Grund. Mit der Literatur des 17. Jahrhunderts mussten sich etwa eine Geistesgeschichte der Literatur oder eine werkimmanente Literaturbetrachtung schwertun, weil beide in den Jahrzehnten vor 1970 dominanten Strömungen sich grosso modo an einem goethezeitlichen Literaturideal orientierten und die Literatur des 17. Jahrhunderts vor diesem Hintergrund als weniger interessant, relevant und wertvoll einstuften. ${ }^{53}$ Am Projekt einer Überwindung dieser Sichtweisen konnte sich eine Sozialgeschichte der Literatur bewähren, und das tat sie in Gestalt bahnbrechender, das Verständnis der Literaturgeschichte des 17. Jahrhunderts wesentlich befördernder Forschungen, in denen sie vorführte, wie man die Literatur der vorgoethezeitlichen Epochen angemessen beschreiben kann, indem man sie vor dem Hintergrund ihrer gesellschaftlichen Kontexte anschaut. ${ }^{54}$ Allerdings hatte es die Barockforschung der 1970er Jahre dann auch schnell mit einem aus diesem Programm resultierenden, bereits länger bekannten Dilemma zu tun: Einerseits kann ich die Literatur des 17. Jahrhunderts nur angemessen verstehen, indem ich ihre gesellschaftlichen Kontexte berücksichtige, andererseits erfahre ich aus der Literatur selbst zunächst einmal wenig über gesellschaftliche Kontexte, weil sie den zeitgenössischen Konventionen entsprechend stark rhetorisch geprägt ist. Eine Lösung dieses Dilem-

53 Vgl. für die Geistesgeschichte etwa Friedrich Gundolf: Martin Opitz. München und Leipzig 1923; ders.: Andreas Gryphius. Heidelberg 1927; für die werkimmanente - oder in diesem Fall genauer: formgeschichtliche - Interpretation exemplarisch Paul Böckmann: Dichterische Gestaltungskräfte in Grimmelshausens Simplicissimus. In: Zeitschrift für deutsche Bildung 18 (1942), S. 226-244.

54 Als wegweisend können etwa gelten Wilfried Barner: Barockrhetorik. Untersuchungen zu ihren geschichtlichen Grundlagen. Tübingen 1970; Klaus Garber: Der locus amoenus und der locus terribilis. Bild und Funktion der Natur in der deutschen Schäfer- und Landlebendichtung des 17. Jahrhunderts. Köln, Wien 1974; Dieter Breuer: Oberdeutsche Literatur 1565-1650. Deutsche Literaturgeschichte und Territorialgeschichte in frühabsolutistischer Zeit. München 1979; Wilhelm Kühlmann: Gelehrtenrepublik und Fürstenstaat. Entwicklung und Kritik des deutschen Späthumanismus in der Literatur des Barockzeitalters. Tübingen 1982. Vgl. dazu in wissenschaftsgeschichtlicher Hinsicht Kai Bremer: Die Frühe Neuzeit - ein Trümmerfeld. Über Anfänge und Ursprünge der Neueren deutschen Literatur. In: Marcel Lepper, Dirk Werle (Hg.): Entdeckung der frühen Neuzeit. Konstruktionen einer Epoche der Literatur- und Sprachgeschichte seit 1750. Stuttgart 2011, S. 39-51. 
mas hatte schon länger darin bestanden zu versuchen, innerhalb des ırhetorischen Zeitalters ${ }^{55}$ Tendenzen einer >realistischen $`$, damit zukunftsweisenden und gleichzeitig Rückschlüsse auf die historische Hintergrundsituation erlaubenden Literatur zu identifizieren. Eine andere bestand darin, die Rhetorizität der Literatur selbst in ihren gesellschaftshistorischen Dimensionen zu analysieren.

Eine Literaturgeschichtsschreibung des 17. Jahrhunderts snach der Kulturgeschichter hat es nach wie vor mit diesem Dilemma zu tun, und das macht ihr die Arbeit nicht leichter in einer Zeit, in der es um die neugermanistische Frühneuzeitforschung fachpolitisch nicht sehr gut bestellt ist: Professuren werden abgeschafft; die Mediävistik macht der Neugermanistik zunehmend das 16. Jahrhundert streitig, das diese weitenteils kampflos preisgibt; im neugermanistischen akademischen Unterricht kommt die Literatur der frühen Neuzeit an vielen universitären Standorten nicht vor. Angesichts dieser Zustände ist es umso wichtiger zu zeigen, was für ein bedeutsames und attraktives Untersuchungsfeld die literaturwissenschaftliche Erforschung der frühen Neuzeit allgemein und des 17. Jahrhunderts im Besonderen ist. Dabei kommt es darauf an, an vorgängige Forschungstraditionen und deren zum Teil bahnbrechende Leistungen anzuknüpfen und ihre großenteils in die richtige Richtung weisenden Bemühungen, eine anspruchsvolle, ideen- wie sozialgeschichtliche Dimensionen berücksichtigende Literaturgeschichtsschreibung der frühen Neuzeit zu entwickeln, weiterzuverfolgen, dabei aber auf der Basis neuerer methodologischer Entwicklungen stetig zu verbessern, etwa in Gestalt einer Gattungsgeschichte als Problemgeschichte, wie sie in diesem Beitrag am Beispiel Christian Ulrich Illenhöfers skizziert wurde.

55 So der Titel des Studienbuchs von Andreas Keller: Frühe Neuzeit. Das rhetorische Zeitalter. Berlin 2008. 